\title{
Pre-Clinical Models and Potential Novel Therapies for Glioblastomas
}

\author{
Michelle Zalles ${ }^{1,2} \bullet$ Rheal A. Towner ${ }^{1,2}$ \\ ${ }^{1}$ Advanced Magnetic Resonance Center, Oklahoma Medical Research Foundation, \\ Oklahoma City, OK, USA; ${ }^{2}$ Oklahoma Center for Neuroscience, University of Oklahoma \\ Health Sciences Center, Oklahoma City, OK, USA
}

Author for correspondence: Rheal A. Towner, Advanced Magnetic Resonance Center, Oklahoma Medical Research Foundation, Oklahoma City, OK, USA.

Email: Rheal-Towner@omrf.org

Doi: https://doi.org/10.36255/exonpublications.gliomas.2021.chapter1

\begin{abstract}
Glioblastoma is an extremely aggressive form of cancer most commonly derived from neural stem cells, astrocytes, and oligodendrocytes that occurs in the brain and has a 5 -year survival rate of $6.7 \%$. These gliomas have an incidence of 3.19 cases per 100,000 person and are assigned grade IV according to the World Health Organization classification of brain tumors. Current approved therapies include surgical resection followed by a combination of radiation and chemotherapy with temozolomide, and more recently tumor-treating (TT) fields. However, there are many limitations with the current treatment strategies due to several resistance mechanisms varying from low concentrations of chemotherapeutics crossing the blood brain barrier to increased risk of temozolomide resistance in a sub-set of patients. In recent years, novel therapies and delivery systems have been developed to overcome these limitations. In this chapter, we discuss pre-clinical assessments and the evaluation of potential, promising therapeutics in xenograft models for glioblastoma using advanced magnetic resonance imaging techniques.
\end{abstract}

Keywords: blood-brain barrier; ELTD1; GL261; OKN-007; pre-clinical mouse models for glioblastoma

In: Gliomas. Debinski W (Editor). Exon Publications, Brisbane, Australia. ISBN: 978-0-6450017-4-7; Doi: https://doi.org/10.36255/exonpublications.gliomas.2021

Copyright: The Authors.

License: This open access article is licenced under Creative Commons Attribution-NonCommercial 4.0 International (CC BY-NC 4.0) https://creativecommons.org/licenses/by-nc/4.0/ 


\section{INTRODUCTION}

Glioblastoma is the most common form of malignant primary brain tumor and accounts for approximately $45.2 \%$ of all malignant primary central nervous system (CNS) tumors (1). These tumors are derived from three cells of origin such as neural stem cells, astrocytes, and oligodendrocytes, and have an annual incidence of 6 per 100,000 diagnosed cases in the United States $(2,3)$. The incidence of glioblastoma increases with age, with patients aged 75-84 having an incidence of 15.03 per 100,000 (4). The average survival for patients is approximately $12-15$ months, and relative survival is extremely low, with less than $5 \%$ of all patients surviving 5 years post-diagnosis, with this value decreasing to $2 \%$ among patients aged 65 years or older (5). Glioblastomas are highly infiltrative and invasive, however, metastasis outside of the CNS is rare. The current treatment method includes surgical resection to remove the bulk of the tumor followed by a combination of chemotherapy with temozolomide (TMZ) or bevacizumab and radiation. Glioblastomas are characterized by their heterogeneity, which poses an important challenge for the development of new drug therapies (5).

To date, no correlation has been found between the development of glioblastoma and smoking or exposure to other carcinogenic agents (4). While the risk factors for glioblastoma development are not well defined, males are predisposed (1.6:1) (1). The most common class of glioblastomas are primary, representing $\sim 90 \%$ of all cases, and most occur in older adults (mean age of 65) without any evidence of precursor lesions (6). Primary glioblastomas are characterized by overexpression of the epidermal growth factor receptor (EGFR), loss of heterozygosity on chromosome 10q, phosphatase and tensin homolog (PTEN) mutations, and lack of isocitrate dehydrogenase 1 (IDH1) mutations (7). EGFR signaling is crucial for the survival, proliferation, migration, and differentiation of all types of cells in the CNS (8). In glioblastomas, EGFR deregulation can be achieved through various mechanisms such as overexpression of the receptor/ ligand and receptor mutation (8). Loss of chromosome 10 is the most frequent genetic alteration (80-90\%) that occurs in glioblastoma. Loss is either entire, or of either the long or the short arm. The PTEN gene is located at 10q23.3 and is crucial for regulating metastasis and invasion (1). This tumor suppressor gene is mutated in $20-40 \%$ of glioblastomas and is most commonly seen in advanced stages of primary glioblastomas $(1,9)$.

On the other hand, secondary glioblastomas ( $10 \%$ of remaining cases) occur in younger patients with a mean age of 45 years, and most commonly develop from lower-grade gliomas (usually astrocytoma or oligodendrogliomas) (1). This type of glioblastoma is characterized by their mutations in TP53, loss of chromosome $1 \mathrm{p} / 19 \mathrm{q}$, as well as IDH1/2 mutations (7). Secondary glioblastomas have also been reported to have longer overall survival when compared to primary (1). TP53 mutations occur in approximately $60-70 \%$ of all secondary glioblastomas, and is a regulator of cell cycle and apoptosis (10). Isocitrate dehydrogenase (IDH) is an enzyme in the Krebs cycle (11). The two forms of IDH (IDHl and IDH2) are NADP-dependent and are most commonly mutated in secondary glioblastomas (10). Various studies have shown how TCA cycle gene mutations contribute to both cancer development and progression (10). IDH1/2 mutations cause an overexpression in both the vascular endothelial growth factor (VEGF) 
and hypoxia-inducible factor $1 \alpha$ (HIF- $1 \alpha$ ) which promotes tumor progression and invasion (10).

\section{THERAPY-LIMITING FACTORS FOR GLIOBLASTOMAS}

There has been little progress made on stand-alone therapeutics in glioblastomas. Numerous clinical trials aimed at treating both primary and recurrent glioblastomas have failed due to various reasons, including ineffectiveness at improving survival and toxicity issues. Heterogeneity continues to be a barrier for therapeutics as each subpopulation of glioblastoma cells have varying degrees of aggressiveness, growth, and therapy resistance (12). In addition to the heterogeneity of the glioblastoma cells, the tumor can be divided into three different regions (Figure 1). The tumor core is described as an area of high proliferation, inflammation, and increased incidence of necrosis (13). Along the boundaries of the tumor core exists the tumor interphase. This area is classified as the transition area between the necrotic core and the outside periphery (13). The interphase has a mild hypoxic environment while maintaining a high proliferation rate (13). Clinicians most commonly try to resect out as much of the tumor core and the interphase as possible, to try to combat glioblastoma (13). However, complete resection of the periphery is difficult as the glioblastoma cells are too diffuse to completely ablate (13). The periphery cells however, have a low proliferation index and have a higher MGMT- cell population that causes these peripheral tumor cells to be more sensitive to TMZ treatment (13).

In addition to the three different regions of the tumor, there are various other challenges to developing effective treatments for glioblastomas. For example,

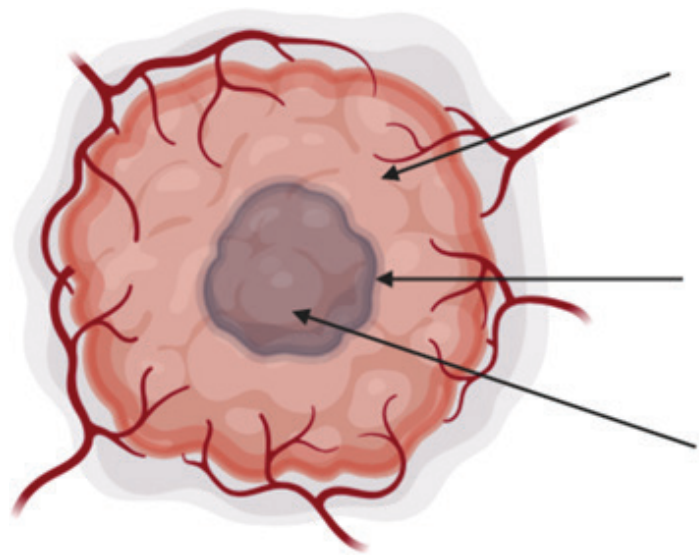

Periphery: Most vascular area of the tumor, some healthy brain tissue with diffuse GBM cells

Interphase: Transition area between the necrotic core and the periphery

Tumor Core: Area of increased

proliferation, inflammation, and necrosis

Figure 1. Description of the tumor. The tumor core is the innermost section of the tumor that is characterized by having a high incidence of necrosis along with increased proliferation and inflammation. The transition area between the tumor core and the periphery is the area known as the interphase. The periphery region includes the healthy brain tissue that has some diffuse glioblastoma cells. (Modified figure from (13); cartoon made with BioRender) 
most gliomas occur in the frontal and temporal lobes $(25.8 \%$ and $19.7 \%$, respectively) while occurrences in the brainstem and the spinal cord are relatively rare (14). The most common symptoms of glioblastomas are loss of vision, numbness, and headaches, usually accompanied by nausea or vomiting (15). These symptoms may be overlooked or mistaken for another disease, which is why glioblastomas are commonly detected in later stages when they begin their widespread infiltration of the brain $(15,16)$. Once the tumors have developed, $60 \%$ of patients experience short seizures, between 2-3 minutes of duration, and suffer from fatigue, confusion, and numbness once the seizure has concluded (16). Neurological deficits such as vision abnormalities, speech problems, and/or loss of motor control, may also be present depending on the location of the tumor (16). Conversely, general symptoms such as personality and mood change may present as primary symptoms, causing patients to take longer to seek medical attention (16).

Another key hurdle in drug development is the blood brain barrier (BBB). This is a highly regulated semipermeable barrier that controls the movement of ions, molecules, and cells between the blood and the CNS (Figure 2). The BBB is crucial in regulating CNS hemostasis in order to protect the CNS from external toxins and pathogens and dysfunction of the BBB leads to ion dysregulation and entry of immune cells that may cause neuronal degradation (17). Endothelial cells form

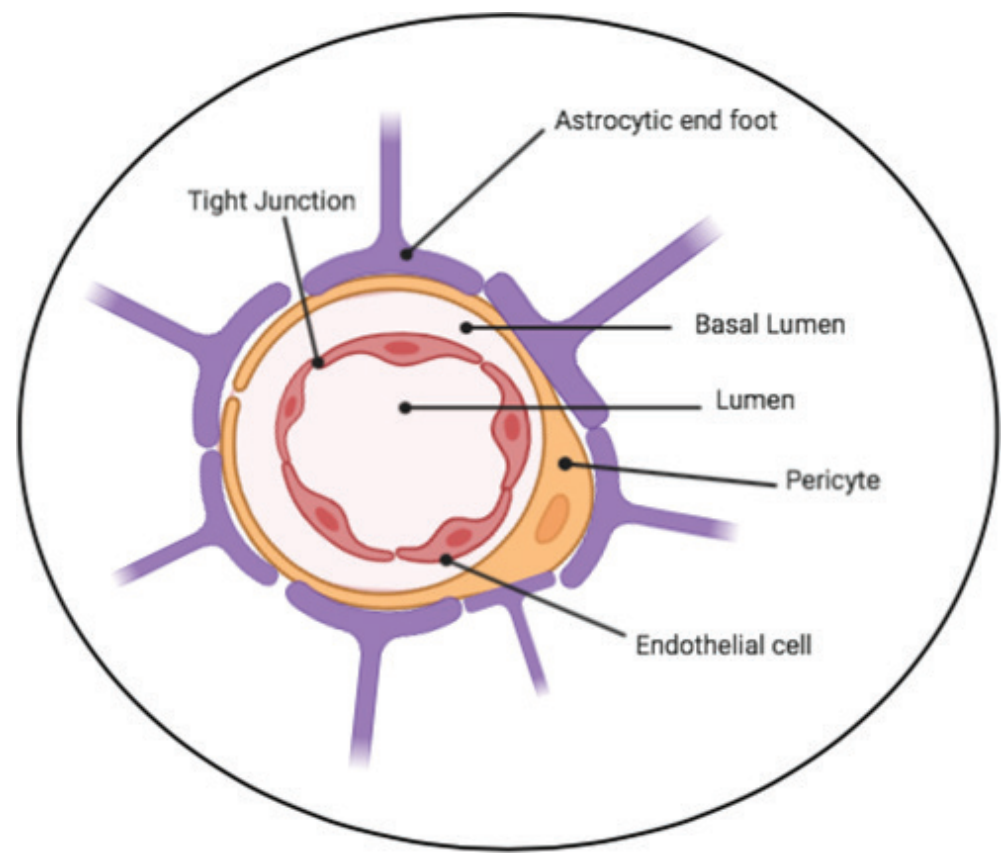

Figure 2. Graphic representation of the BBB. The endothelial cells line the lumen to form the BBB. The pericytes (shown in orange) and astrocytic feet (shown in purple) are key in keeping the tight structure of the BBB. The cartoon is modified from a BioRender BBB template. 
the BBB and are connected via tight junctions (17). Pericytes are important for regulating vascular function and vessel remodeling (18). In addition, pericytes and astrocytic feet help maintain the structure and rigidity of the BBB; pericyte-deficient mutant mice were shown to have increased BBB permeability as opposed to wild-type $(18,19)$. Astrocytic feet wrap around and cover the majority of the outermost surface area of the BBB, and in addition to aiding in the structure of the $\mathrm{BBB}$, they are also crucial in regulating the signaling pathways that help retain the junction complexes such as the tight junctions (18). There are various factors, such as lipophilicity, molecular weight and charge that influence a molecule's capacity to permeate the $\mathrm{BBB}(17,18)$.

Brain tumors, such as glioblastomas, can disrupt the integrity of the BBB which increases vessel permeability allowing immune cells, plasma, and fluid to leak into the tumor regions. In glioblastoma, BBB disruption is most commonly determined via contrast-enhanced magnetic resonance imaging (MRI) by administering gadolinium-based contrast agents. This hydrophilic contrast agent accumulates within the extracellular space, and this area is then enhanced on Tl-weighted (T1W) images (20). Additionally, regions of non-enhancing edema that show dysfunction or permeability of the BBB can be detected by T2-weighted (T2W) or T2 fluid attenuated inversion recovery (FLAIR) MRI (20). However, while T1/2 weighted images may show the general qualitative analysis of the BBB, they fail to show the true extent to which the BBB is disrupted in each patient, as it can vary both from patient to patient and from region to region in the same tumor (20).

\section{PRECLINICAL MODELS}

Although there is a clinical treatment plan in place, the prognosis for glioblastoma is still dismal. Preclinical mouse models are necessary to study the biology of this tumor and further identify and evaluate new potential therapies (21). Mice are most commonly used for glioblastoma research due to their accessibility and lower cost. Additionally, it is relatively easy to genetically manipulate mice, and they have short breeding times (22). Currently, glioblastoma preclinical mouse models are classified into three main categories: xenografts, genetically engineered (GEMMs), and syngeneic mouse models (Figure 3).

In xenograft mouse models, human tumor cells are transplanted either subcutaneously, or orthotopically into the brain with stereotactic surgery. Although subcutaneous injections have been widely used, these tumors lack the brain microenvironment, and therefore cannot be used to fully study the behavior of the disease (22). On the other hand, cells that are transplanted into the organ of origin allow for proper brain infiltrative behavior. Another advantage of orthotopic xenografts is that tumor sizes and sites are more consistent (22). The human tumor cells are transplanted into immunocompromised mice to ensure that the mouse does not reject the human cells (23). Some common and readily accepted immunocompromised mice are athymic nude mice, severely compromised immunodeficient mice (SCID), or non-obese diabetic (NOD)/SCID mice. Athymic nude mice are genetically modified to either have a deteriorated or absent thymus. This causes the animal to be unable to produce mature $\mathrm{T}$ cells, and have a reduced number of circulating lymphocytes (24). SCID mice are commonly used to 


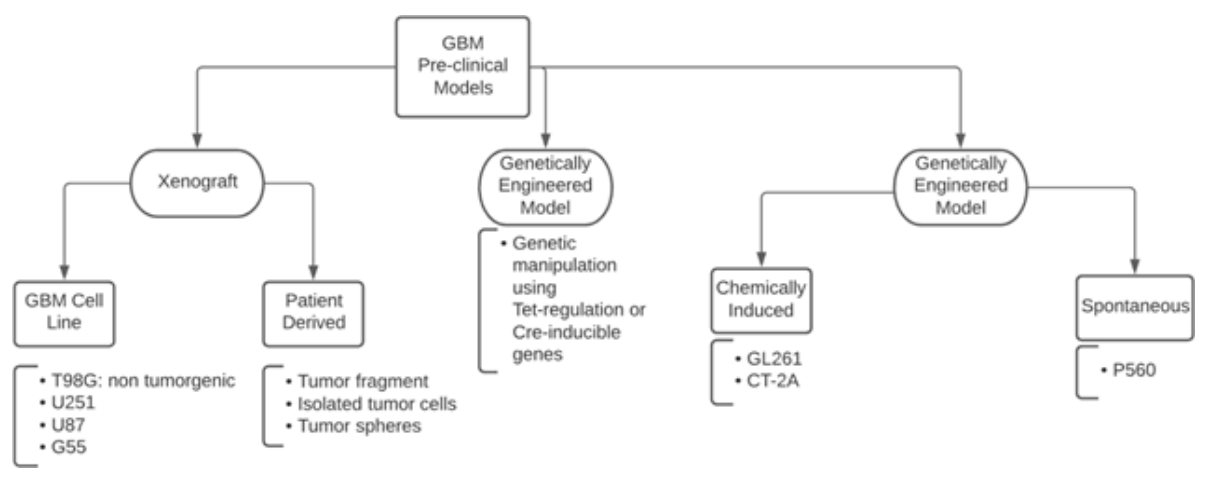

Figure 3. Summary of GBM Pre-clinical Models. A breakdown of the different classifications of the common GBM pre-clinical models found in this chapter.

research various cancers along with other human diseases because SCID mice lack B and T cells in the thymus, spleen, and lymph nodes (25). In addition to the SCID background, NOD/SCID mice have deficient natural killer cell function (26).

The xenografts can be further subclassified into either glioblastoma cell-line or patient-derived xenografts. The results of drug therapy studies from both glioblastoma cell-line or patient-derived xenografts can be obtained in weeks. Glioblastoma cell-lines are commercially available, and usually have high engraftment and growth rates (21). Additionally, glioblastoma cell-lines have higher predictability and more reliable growth/progression of the tumors. Some of the most commonly used glioblastoma cell-lines for in vitro and in vivo research include T98G, U251, and U87. The T98G cell line was generated from a 61-year old male and is characterized as having a high expression of actin alpha 2 (ACTA2) which is key for cell motility (27). However, T98G cells are not tumorigenic in mice (27).

Both the U251 and U87 established cell lines are commercially available (American Type Culture Collection-ATTC). The U251 cell line was generated from a 75-year old male with astrocytoma, and the U87 cell line was obtained from a 44-year old female $(28,29)$. Both of these cell lines were generated in the 1960s $(28,29)$. Hematoxylin and eosin analysis of U251 tumor tissue has shown characteristic tumor cell infiltrative pattern and areas of necrosis similar to that seen in human glioblastoma (30). Additionally, U251 tumors have high cell proliferation levels with over 50\% Ki-67 positivity staining (31). Although the U87 cell line is one of the most widely used models with over 2,000 entries in PubMed over the last 5 years, the U87MG cells have other characteristics that are not commonly associated with glioblastomas such as having non-diffuse infiltrative patterns (30). In fact, when the U87MG cell line (obtained from ATCC) is compared to the original tumor, the DNA profile is drastically different (32). This suggests that the U87MG cell line commonly used today is a completely different version as the original tumor taken in the 1960s (32).

This begs the question, why continue using glioblastoma cell-lines if they do not mimic the true biological nature of glioblastomas? Although the U87 cell line 
is not characteristic of glioblastomas, there are other glioblastoma cell lines that still remain true to the disease. For example, a less commonly used cell line, with only 33 entries in PubMed.gov (National Institutes of Health National Library of Medicine) in the past 5 years, is the human G55 cell line. These cells were originally taken from a 65-year-old male with an anaplastic astrocytoma and have displayed both tumorgenicity in nude mice and classic glioblastoma behavior with respect to aggressive proliferation, angiogenesis, and migration $(33,34)$.

Patient derived orthotopic xenograft (PDOX) models are created from direct implantation of either biopsied tumor fragments or freshly isolated cells without the need for intermediate cell culture (22). These models retain the genetic, histological, and molecular profile similar to the primary tumors, even after multiple cell passages $(21,22)$. However, a disadvantage is that PDOX models are costly and require fresh tumor fragments/cells, therefore limiting the number of facilities that can use these models. In addition to PDOX, other patient derived xenografts can be created by injecting glioblastoma tumor spheres, also known as neurospheres. Neurospheres are produced by cultivating neural stem-like cells from primary brain tumors that can then be transplanted into immunodeficient mice to form gliomas $(35,36)$. These tumor spheres also have the ability to retain tumor heterogeneity and specific aspects of tumor growth, such as tumor angiogenesis and cell invasion, similar to the patient's original tumor $(37,38)$. However, a disadvantage of this model is that not all tumors are able to be successfully cultured as tumor spheres.

GEMMs, on the other hand, have their genetic profile altered to express one or several mutated, deleted, or overexpressed genes that contribute to the malignancy that better recapitulate the molecular evolution of GBMs (23). Commonly, the GEMMs that have been established use a combination of tumor suppressor inactivation ( 553 and/or $\mathrm{Rb}$ ) and the activation of receptor tyrosine kinases (RTK) and $\mathrm{Rb}$ signaling, most commonly though PTEN or Nf1 deletion (39). For example, an established glioma model uses a conventional knockout of Nf1 and p53 to develop tumors is approximately $92 \%$ of the animals by 6 months (40). It is key to note that some of the mutations in key genes are lethal and therefore some GEMMs must be generated using Tet-regulation of Cre-inducible gene alleles in order to express/inactivate certain genes at specific time points (22). The tumors that result from GEM models are usually composed of homogeneous genetic change and therefore, do not reflect the heterogeneity of human glioblastoma (21). However, these models are extremely useful in determining and identifying various molecular events thought to be key in tumor growth and progression by manipulating particular pathways that drive brain tumor initiation. GEMMs are also an ideal system to study the effects of particular drugs on their molecular targets in an immunocompetent host. A host with an intact immune system allows for proper analysis of the interaction of the tumor microenvironment and potential therapeutics. Furthermore, GEMMs can be easily used in humanized mice studies. A major disadvantage however, is that GEM models commonly require months to a year to develop prior to starting drug therapy studies (23). Additionally, because the tumors are not of human origin, they cannot reliably mimic glioblastoma behavior and cannot reliably predict therapeutic response (23). A comprehensive description of mouse models of experimental glioblastoma is provided in chapter 2. 
Lastly, syngeneic mouse models are also commonly used in glioblastoma research, due to the fact the cells can be implanted in immunocompetent animals. This model allows for proper examination of immune interactions and is commonly used to study potential immunotherapeutics (21). Within syngeneic mouse models, there are two different sub-categories: carcinogen/chemically induced and spontaneous. Carcinogen induced models include GL261 and CT-2A. GL261 was the first and is the most commonly used immunocompetent mouse model. These cells can be implanted and form tumors either in the subcutaneous region or intracranially (41). However, there is some concern that the GL261model has also drifted genetically and does not authentically model glioblastoma characterizations or attributes. Examination of GL261 characteristics showed a mutation in KRAS, an oncogene mutated in $25 \%$ of all tumors, and may affect how different therapeutics respond (41). Additionally, for the CT-2A models, 20-methylcholanthrene pellets were intracranially implanted into C57/BL6 mice and the subsequent astrocytoma formed was used to establish the cell line (42). Similar to classical GBM characteristics, CT-2A tumors are deficient in PTEN protein, are necrotic and chemo-resistant, and undergo unregulated angiogenesis (38). While spontaneous models, such as P560, best reflect the natural spontaneous nature of human GBM disease, large cohorts of animal are necessary, and the cost may outweigh the positives (38).

With advancements in science, we can now monitor the transplanted tumor cells and tumor growth in vivo. One way to achieve this is by expressing a luciferase cassette in the tumor cells so that movement of the cells and growth of the tumor can be monitored by bioluminescence. However, a disadvantage to this method is that it commonly requires a larger number of cells to be injected, which may have an impact on the tissue architecture (22). Another method is MRI, which allows monitoring of tumor growth and progression, along with various other aspects of tumorigenesis such as perfusion imaging.

Pre-clinical mouse models have been widely used for many years. Ideal models should mimic classical histopathology, behavior, and genetic mutations as human glioblastomas as well as be reproducible. However, to date, no one model is able to completely mimic all characteristics of glioblastoma. This is because ongoing research uncovers and updates genetic and cellular mechanisms in human glioblastomas. Additionally, continuous passaging of cell lines/PDX models introduces new mutations and genetic drift over time. Therefore, further research needs to be done to establish mouse models that can fully represent human glioblastoma.

\section{PROMISING THERAPEUTICS}

To date, the current treatment plan is not effective in combating human glioblastomas. Although animals have been successfully treated with bevacizumab, as well as check-point inhibitors, the results did not translate to significant increase of survival in patients. Therefore, new therapeutic approaches are crucial. While there are various therapies being examined for glioblastomas, this chapter touches on only three promising therapies from the literature.

EGFR/HERl in humans is a transmembrane receptor tyrosine kinase that is overexpressed in various cancers, including glioblastoma (43). Overexpression of 
EGFR results in increased unregulated growth and survival of glioblastoma cells (44). While the EGFR has been an attractive drug target against glioblastomas for years, there has not been an effective therapy reported. Both firstand second-generation EGFR inhibitors (gefitinib and afatinib, respectively) successfully decreased cell proliferation, growth and angiogenesis in tumor models, however, did not have a significant effect in clinical trials (43). More specifically, gefitinib did not show an effect on survival in phase II trials of relapsed glioblastoma, and afatinib did not have an effect on survival in primary nor recurrent glioblastomas $(45,46)$. The failure of the first- and second- generation of inhibitors exposed the two main issues with the drug. First, in order for the drug to have an effect, sufficiently high concentrations of the drug are required (43). Secondly, the EGFR inhibitors are unable to successfully cross the BBB (43). As stated above, the BBB of patients is not fully disrupted, and the extent of disruption differs from patient to patient and from region to region within the same tumor (43). Therefore, in order for an EGFR inhibitor to be successful, the new drug must penetrate the BBB. Osimertinib (AZD9291) is currently used to treat lung cancer and is an oral, third generation irreversible EGFR inhibitor $(43,47)$. Studies have shown that AZD9291 can penetrate the BBB, inhibit tumor growth, and may be effective as a brain tumor therapeutic (47). When comparing AZD9291 to gefitinib, the concentration of AZD9291 in the brain can reach up to 10-fold higher than gefitinib (43). Tumor heterogeneity is also an important factor to consider when targeting tumors.

Unregulated angiogenesis is critical for the maintenance of the tumor, as the newly formed blood vessels deliver nutrients deep inside the tumor core. To date, the main angiogenic pathways have been Notch/DLL4 and the vascular endothelial growth factor (VEGF). The notch signaling pathways have been highly conserved throughout various species and are crucial for multiple aspects of tumorigenesis (cell proliferation, migration, and tumor angiogenesis) $(48,49)$. Similarly, VEGF promotes tumorigenesis and angiogenesis (34). The epidermal growth factor, latrophilin and seven transmembrane domain-containing protein 1 (ELTD1, alternatively known as ADGRL4) is an angiogenic biomarker. In normal vasculature, the expression of ELTD1 is increased by VEGF, and decreased by the Notch/DLL4 signaling pathway (50). ELTDl is overexpressed in human highgrade gliomas, when compared to low grade gliomas, and when inhibited through varying antibodies (polyclonal, monovalent monoclonal, and single chain variable fragment ( $\mathrm{scFv})$ ) effectively decrease tumor volumes, completely normalize the tumor associated vasculature and increase survival $(34,51,52)$. RNA-sequencing analysis also revealed that anti-ELTD1 therapy may have an effect on other aspects of tumorigenesis such as migration, cell proliferation, and invasion (34). While the BBB has been an issue for some potential therapies, molecular targeted MRI showed that an optimized scFv antibody treatment against ELTDl was successful in reaching extremely diffuse tumor regions that were otherwise undetectable via conventional MRI (51). In a G55 xenograft mouse model, anti-ELTD1 treatment was also found to reduce and target two angiogenic pathways, VEGFR2 and Notch $(34,51,52)$. Additionally, unlike Bevacizumab, an anti-angiogenic therapy, antiELTD1 treatment had no signs of hemorrhaging in a pre-clinical mouse model (34). Further analysis is needed to determine if anti-ELTDl therapy would be effective in clinical trials. Ongoing research in our group is assessing the ability of antiELTD1 antibody therapy against other aspects of tumorigenesis (e.g. cell 
proliferation, cell invasion and apoptosis), as suggested from RNA-seq data in our previous pre-clinical study (34).

OKN-007 (OKN) is a small molecule that can cross the BBB and specifically affects the transforming growth factor $\beta 1$ (TGF $\beta 1$ ) (53). OKN has been widely studied in various glioma models (C6, U87, F98, GL261, and G55) and is currently in two glioblastoma clinical trials. The first is a phase II clinical trial of OKN-007 combined with TMZ in patients that have recurrent glioblastoma, while the second is an early phase I trial that looks at the side effects of OKN-007 with TMZ in patients that are undergoing concomitant radiotherapy after surgery (54). OKN-007 significantly affects every aspect of tumorigenesis by decreasing cellular proliferation, migration, angiogenesis, and increasing apoptosis (55-57). Treatment with OKN resulted in increased survival, decreased tumor volumes (as measured by MRI), and inhibition of tumor necrosis (as measured by MRI morphological and diffusion-weighted imaging, MR spectroscopy, and histology) (57-59). Regarding angiogenesis, OKN decreased both VEGFR2 and HIF-l $\alpha$ protein expression $(56,59)$. Additionally, OKN and TMZ combination treatment significantly increased percent survival, decreased tumor volumes, and normalized the vasculature in TMZ resistant glioblastoma cell lines (55). RNA-sequencing studies have also shown that OKN-007 also has an effect on 57 genes associated with the extracellular matrix through TGF $\beta 1$ including collagens and MMPs (55). Altogether, this suggests that OKN-007 may be effective in targeting multiple aspects of tumorigenesis. To date, no dose-limiting toxicities, nor adverse side effects, were observed with OKN-007 in an initial phase Ib/IIa clinical trial (60). Currently, OKN-007 is being investigated in an ongoing multi-institutional phase II clinical trial.

\section{CONCLUSION}

There have been numerous drug therapies proposed for the treatment of glioblastoma. However, many of those proposed therapies have failed due to various reasons, ranging from inability to penetrate the $\mathrm{BBB}$ to failure to translate significant results in human trials. Here we discussed three different promising therapies that are able to bypass the BBB and have the ability to hit multiple tumorigenic pathways. Glioblastomas are complex heterogeneous tumors that have the ability to adapt and build resistance against existing treatments. This therefore prompts the need for new therapies that have the capacity to target various pathways so that they remain effective against all subpopulations in the tumor.

Conflict of Interest: Dr. Towner holds patents regarding the use of OKN-007 and anti-ELTDl antibody therapy in gliomas. Ms. Zalles declares no potential conflicts of interest with respect to research, authorship, and/or publication of this chapter.

Copyright and Permission Statement: The authors confirm that the materials included in this chapter do not violate copyright laws. Where relevant, appropriate permissions have been obtained from the original copyright holder(s), and all original sources have been appropriately acknowledged or referenced. 


\section{REFERENCES}

1. Thakkar JP, Dolecek TA, Horbinski C, Ostrom QT, Lightner DD, Barnholtz-Sloan JS, et al. Epidemiologic and molecular prognostic review of glioblastoma. Cancer Epidemiol Biomarkers Prev. 2014;23(10):1985-96. https://doi.org/10.1158/1055-9965.EPI-14-0275

2. Nayak L, Reardon DA. High-grade Gliomas. Continuum. Neuro-oncology.2017;23(6):1548-63. https://doi.org/10.1212/CON.0000000000000554

3. Yao M, Li S, Wu X, Diao S, Zhang G, He H, et al. Cellular origin of glioblastoma and its implication in precision therapy. Cell Mol Immunol. 2018;15(8):737-9. https://doi.org/10.1038/cmi.2017.159

4. Tamimi AF, Juweid M. Epidemiology and Outcome of Glioblastoma. In: De Vleeschouwer S, editor. Glioblastoma. Brisbane (AU)2017. https://doi.org/10.15586/codon.glioblastoma.2017.ch8

5. Eder K, Kalman B. Molecular heterogeneity of glioblastoma and its clinical relevance. Pathol Oncol Res. 2014;20(4):777-87. https://doi.org/10.1007/s12253-014-9833-3

6. Olar A, Aldape KD. Using the molecular classification of glioblastoma to inform personalized treatment. J Pathol. 2014;232(2):165-77. https://doi.org/10.1002/path.4282

7. Ohgaki H, Kleihues P. The definition of primary and secondary glioblastoma. Clin Cancer Res. 2013;19(4):764-72. https://doi.org/10.1158/1078-0432.CCR-12-3002

8. Huang $\mathrm{PH}, \mathrm{Xu} \mathrm{AM}$, White FM. Oncogenic EGFR signaling networks in glioma. Sci Signal. 2009;2(87):re6. https://doi.org/10.1126/scisignal.287re6

9. Wirsching HG, Galanis E, Weller M. Glioblastoma. Handb Clin Neurol. 2016;134:381-97. https:// doi.org/10.1016/B978-0-12-802997-8.00023-2

10. Ohgaki H, Kleihues P. Genetic pathways to primary and secondary glioblastoma. Am J Pathol. 2007;170(5):1445-53. https://doi.org/10.2353/ajpath.2007.070011

11. Huang J, Yu J, Tu L, Huang N, Li H, Luo Y. Isocitrate Dehydrogenase Mutations in Glioma: From Basic Discovery to Therapeutics Development. Front Oncol. 2019;9:506. https://doi.org/10.3389/ fonc. 2019.00506

12. Harder BG, Blomquist MR, Wang J, Kim AJ, Woodworth GF, Winkles JA, et al. Developments in Blood-Brain Barrier Penetrance and Drug Repurposing for Improved Treatment of Glioblastoma. Front Oncol. 2018;8:462. https://doi.org/10.3389/fonc.2018.00462

13. Persano L, Rampazzo E, Della Puppa A, Pistollato F, Basso G. The three-layer concentric model of glioblastoma: cancer stem cells, microenvironmental regulation, and therapeutic implications. Scientific World J. 2011;11:1829-41. https://doi.org/10.1100/2011/736480

14. Ostrom QT, Gittleman H, Liao P, Rouse C, Chen Y, Dowling J, et al. CBTRUS statistical report: primary brain and central nervous system tumors diagnosed in the United States in 2007-2011. Neuro Oncol. 2014;16 Suppl 4:ivl-63. https://doi.org/10.1093/neuonc/nou223

15. Alexander BM, Cloughesy TF. Adult Glioblastoma. J Clin Oncol. 2017;35(21):2402-9. https://doi. org/10.1200/JCO.2017.73.0119

16. Davis ME. Glioblastoma: Overview of Disease and Treatment. Clin J Oncol Nurs. 2016;20(5 Suppl): S2-8. https://doi.org/10.1188/16.CJON.S1.2-8

17. Daneman R, Prat A. The blood-brain barrier. Cold Spring Harb Perspect Biol. 2015;7(1):a020412. https://doi.org/10.1101/cshperspect.a020412

18. Arvanitis CD, Ferraro GB, Jain RK. The blood-brain barrier and blood-tumour barrier in brain tumours and metastases. Nat Rev Cancer. 2020;20(1):26-41. https://doi.org/10.1038/s41568-019-0205-x

19. Armulik A, Genove G, Mae M, Nisancioglu MH, Wallgard E, Niaudet C, et al. Pericytes regulate the blood-brain barrier. Nature. 2010;468(7323):557-61. https://doi.org/10.1038/nature09522

20. Sarkaria JN, Hu LS, Parney IF, Pafundi DH, Brinkmann DH, Laack NN, et al. Is the blood-brain barrier really disrupted in all glioblastomas? A critical assessment of existing clinical data. Neuro Oncol. 2018;20(2):184-91. https://doi.org/10.1093/neuonc/nox175

21. Kijima N, Kanemura Y. Mouse Models of Glioblastoma. In: Glioblastoma. Vleeschouwer SD, editor. Codon Publications 2017. http://dx.doi.org/10.15586/codon.glioblastoma.2017

22. Robertson FL, Marques-Torrejon MA, Morrison GM, Pollard SM. Experimental models and tools to tackle glioblastoma. Dis Model Mech. 2019;12(9). https://doi.org/10.1242/dmm.040386 
23. Richmond A, Su Y. Mouse xenograft models vs GEM models for human cancer therapeutics. Dis Model Mech. 2008;1(2-3):78-82. https://doi.org/10.1242/dmm.000976

24. Dubey M. Nude Mice The Jackson Laboratory [Available from: https://www.jax.org/news-andinsights/jax-blog/2019/december/nude-mice. [Accessed on 16 March 2021]

25. Charles River SCID Mice. https://www.criver.com/products-services/research-models-services/animalmodels/mice/immunodeficient-mice/scid-mice?region=3616. [Accessed on 16 March 2021]

26. Charles River NOD SCID Mouse [Available from: https:/www.criver.com/products-services/findmodel/nod-scid-mouse?region=3616. [Accessed on 16 March 2021]

27. Jacobs VL, Valdes PA, Hickey WF, De Leo JA. Current review of in vivo GBM rodent models: emphasis on the CNS-1 tumour model. ASN Neuro. 2011;3(3):e00063. https://doi.org/10.1042/AN20110014

28. Westermark B, Ponten J, Hugosson R. Determinants for the establishment of permanent tissue culture lines from human gliomas. Acta Pathol Microbiol Scand A. 1973;81(6):791-805. https://doi. org/10.1111/j.1699-0463.1973.tb03573.x

29. Ponten J, Macintyre EH. Long term culture of normal and neoplastic human glia. Acta Pathol Microbiol Scand. 1968;74(4):465-86. https://doi.org/10.1111/j.1699-0463.1968.tb03502.x

30. Candolfi M, Curtin JF, Nichols WS, Muhammad AG, King GD, Pluhar GE, et al. Intracranial glioblastoma models in preclinical neuro-oncology: neuropathological characterization and tumor progression. J Neurooncol. 2007;85(2):133-48. https://doi.org/10.1007/s11060-007-9400-9

31. Radaelli E, Ceruti R, Patton V, Russo M, Degrassi A, Croci V, et al. Immunohistopathological and neuroimaging characterization of murine orthotopic xenograft models of glioblastoma multiforme recapitulating the most salient features of human disease. Histol Histopathol. 2009;24(7):879-91.

32. Allen M, Bjerke M, Edlund H, Nelander S, Westermark B. Origin of the U87MG glioma cell line: Good news and bad news. Sci Transl Med. 2016;8(354):354re3. https://doi.org/10.1126/scitranslmed.aaf6853

33. Westphal M, Hansel M, Hamel W, Kunzmann R, Holzel F. Karyotype analyses of 20 human glioma cell lines. Acta Neurochir (Wien). 1994;126(1):17-26. https://doi.org/10.1007/BF01476489

34. Zalles M, Smith N, Ziegler J, Saunders D, Remerowski S, Thomas L, et al. Optimized monoclonal antibody treatment against ELTD1 for GBM in a G55 xenograft mouse model. J Cell Mol Med. 2020;24(2):1738-49. https://doi.org/10.1111/jcmm.14867

35. Johnson S, Chen H, Lo PK. In vitro Tumorsphere Formation Assays. Bio Protoc. 2013;3(3). https:// doi.org/10.21769/BioProtoc.325

36. Laks DR, Masterman-Smith M, Visnyei K, Angenieux B, Orozco NM, Foran I, et al. Neurosphere formation is an independent predictor of clinical outcome in malignant glioma. Stem Cells. 2009;27(4):980-7. https://doi.org/10.1002/stem.15

37. Wakimoto H, Mohapatra G, Kanai R, Curry WT, Jr., Yip S, Nitta M, et al. Maintenance of primary tumor phenotype and genotype in glioblastoma stem cells. Neuro Oncol. 2012;14(2):132-44. https:// doi.org/10.1093/neuonc/nor195

38. Gunther HS, Schmidt NO, Phillips HS, Kemming D, Kharbanda S, Soriano R, et al. Glioblastomaderived stem cell-enriched cultures form distinct subgroups according to molecular and phenotypic criteria. Oncogene. 2008;27(20):2897-909. https://doi.org/10.1038/sj.onc.1210949

39. Simeonova I, Huillard E. In vivo models of brain tumors: roles of genetically engineered mouse models in understanding tumor biology and use in preclinical studies. Cell Mol Life Sci. 2014;71(20):4007-26. https://doi.org/10.1007/s00018-014-1675-3

40. Reilly KM, Loisel DA, Bronson RT, McLaughlin ME, Jacks T. Nf1;Trp53 mutant mice develop glioblastoma with evidence of strain-specific effects. Nat Genet. 2000;26(1):109-13. https://doi. org/10.1038/79075

41. Szatmari T, Lumniczky K, Desaknai S, Trajcevski S, Hidvegi EJ, Hamada H, et al. Detailed characterization of the mouse glioma 261 tumor model for experimental glioblastoma therapy. Cancer Sci. 2006;97(6):546-53. https://doi.org/10.1111/j.1349-7006.2006.00208.x

42. Binello E, Qadeer ZA, Kothari HP, Emdad L, Germano IM. Stemness of the CT-2A Immunocompetent Mouse Brain Tumor Model: Characterization In Vitro. J Cancer. 2012;3:166-74. https://doi. org/10.7150/jca.4149 
43. Liu X, Chen X, Shi L, Shan Q, Cao Q, Yue C, et al. The third-generation EGFR inhibitor AZD9291 overcomes primary resistance by continuously blocking ERK signaling in glioblastoma. J Exp Clin Cancer Res. 2019;38(1):219. https://doi.org/10.1186/s13046-019-1235-7

44. Padfield E, Ellis HP, Kurian KM. Current Therapeutic Advances Targeting EGFR and EGFRvIII in Glioblastoma. Front Oncol. 2015;5:5. https://doi.org/10.3389/fonc.2015.00005

45. Chakravarti A, Wang M, Robins HI, Lautenschlaeger T, Curran WJ, Brachman DG, et al. RTOG 0211: a phase $1 / 2$ study of radiation therapy with concurrent gefitinib for newly diagnosed glioblastoma patients. Int J Radiat Oncol Biol Phys. 2013;85(5):1206-11. https://doi.org/10.1016/j. ijrobp.2012.10.008

46. Reardon DA, Nabors LB, Mason WP, Perry JR, Shapiro W, Kavan P, et al. Phase I/randomized phase II study of afatinib, an irreversible ErbB family blocker, with or without protracted temozolomide in adults with recurrent glioblastoma. Neuro Oncol. 2015;17(3):430-9. https://doi.org/10.1093/ neuonc/noul60

47. Cross DA, Ashton SE, Ghiorghiu S, Eberlein C, Nebhan CA, Spitzler PJ, et al. AZD9291, an irreversible EGFR TKI, overcomes T790M-mediated resistance to EGFR inhibitors in lung cancer. Cancer Discov. 2014;4(9):1046-61. https://doi.org/10.1158/2159-8290.CD-14-0337

48. Li J, Li Q, Lin L, Wang R, Chen L, Du W, et al. Targeting the Notchl oncogene by miR-139-5p inhibits glioma metastasis and epithelial-mesenchymal transition (EMT). BMC Neurol. 2018;18(1):133. https://doi.org/10.1186/s12883-018-1139-8

49. Gersey Z, Osiason AD, Bloom L, Shah S, Thompson JW, Bregy A, et al. Therapeutic Targeting of the Notch Pathway in Glioblastoma Multiforme. World Neurosurg. 2019;131:252-63 e2. https://doi. org/10.1016/j.wneu.2019.07.180

50. Masiero M, Simoes FC, Han HD, Snell C, Peterkin T, Bridges E, et al. A core human primary tumor angiogenesis signature identifies the endothelial orphan receptor ELTD1 as a key regulator of angiogenesis. Cancer Cell. 2013;24(2):229-41. https://doi.org/10.1016/j.ccr.2013.06.004

51. Zalles M, Smith N, Saunders D, Saran T, Thomas L, Gulej R, et al. Assessment of an scFv Antibody Fragment Against ELTDl in a G55 Glioblastoma Xenograft Model. Transl Oncol. 2020;13(3): 100737. https://doi.org/10.1016/j.tranon.2019.12.009

52. Ziegler J, Zalles M, Smith N, Saunders D, Lerner M, Fung KM, et al. Targeting ELTD1, an angiogenesis marker for glioblastoma (GBM), also affects VEGFR2: molecular-targeted MRI assessment. Am J Nucl Med Mol Imaging. 2019;9(1):93-109.

53. Towner RA, Zalles M, Saunders D, Smith N. Novel approaches to combat chemoresistance against glioblastomas. Cancer Drug Resist. 2020;3: https://doi.org/10.20517/cdr.2020.38

54. Institute NC. Clinical Trials Using PBN Derivative OKN-007: NIH; 2020 [Available from: https:// www.cancer.gov/about-cancer/treatment/clinical-trials/intervention/pbn-derivative-okn-007].

55. Towner RA, Smith N, Saunders D, Brown CA, Cai X, Ziegler J, et al. OKN-007 Increases temozolomide (TMZ) Sensitivity and Suppresses TMZ-Resistant Glioblastoma (GBM) Tumor Growth. Transl Oncol. 2019;12(2):320-35. https://doi.org/10.1016/j.tranon.2018.10.002

56. Towner RA, Gillespie DL, Schwager A, Saunders DG, Smith N, Njoku CE, et al. Regression of glioma tumor growth in F98 and U87 rat glioma models by the Nitrone OKN-007. Neuro Oncol. 2013;15(3):330-40. https://doi.org/10.1093/neuonc/nos337

57. de Souza PC, Balasubramanian K, Njoku C, Smith N, Gillespie DL, Schwager A, et al. OKN-007 decreases tumor necrosis and tumor cell proliferation and increases apoptosis in a preclinical F98 rat glioma model. J Magn Reson Imaging. 2015;42(6):1582-91. https://doi.org/10.1002/jmri.24935

58. Coutinho de Souza P, Smith N, Atolagbe O, Ziegler J, Njoku C, Lerner M, et al. OKN-007 decreases free radical levels in a preclinical F98 rat glioma model. Free Radic Biol Med. 2015;87:157-68. https://doi.org/10.1016/j.freeradbiomed.2015.06.026

59. de Souza PC, Smith N, Pody R, He T, Njoku C, Silasi-Mansat R, et al. OKN-007 decreases VEGFR-2 levels in a preclinical GL261 mouse glioma model. Am J Nucl Med Mol Imaging. 2015;5(4):363-78.

60. Battiste JD, Ikeguchi A, Woo S, Sharan S, Zhao YD, Cohoon A, et al. Phase Ib clinical trial of OKN-007 in recurrent malignant glioma. Journal of Clinical Oncology. 2020;38(15_suppl):2538-. https://doi. org/10.1200/JCO.2020.38.15_suppl.2538 
